\title{
Thin film optical BTB pH sensors using sol-gel method in presence of surfactants
}

\author{
Issa M El Nahhal', Shehata M Zourab, Fawzi S Kodeh and Alaa I Qudaih
}

\begin{abstract}
Bromothymol blue (BTB) thin films were prepared by depositing a thin layer of a solution containing tetraorthosilicate and $\mathrm{BTB} \mathrm{pH}$ indicator in the presence of surfactants, namely cetyltrimethylammonium bromide $\left(\mathrm{C}_{19} \mathrm{H}_{42} \mathrm{BrN}, \mathrm{CTAB}\right)$ or dodecyldimethylamine oxide $\left(\mathrm{C}_{14} \mathrm{H}_{31} \mathrm{NO}, 69 \%\right), \mathrm{C}_{16} \mathrm{H}_{35} \mathrm{NO}(11 \%)$, Genaminox LA onto a glass slide substrate. CTAB or GLA surfactants were used to improve the mesostructure of the host material and to increase its porosity. Optical BTB thin films were found to be sensitive against $\mathrm{pH}$ and behave as free BTB. This provides evidence for weak interactions between the BTB molecules and the host silica matrix. The effects of the sol mixture on the stability and reproducibility and the colorimetric response to solutions of different pH were examined and pKa values were determined. The BTB thin film sensors showed an advantage over the encapsulated monolithic sensors in which the thin film sensors exhibit a faster response time than the monolithic disks. These pH sensors showed reproducibility and high stability behavior.
\end{abstract}

Keywords: Bromothymol blue, Optical thin films, pH sensors, Surfactants, Tetraorthosilicate

\section{Background}

The sol-gel process is used to immobilize $\mathrm{pH}$ indicators in the silica matrix network through hydrolysis and subsequent polycondensation of silicon alkoxide precursors at room temperature (RT). The sol-gel matrix provides a physical and chemical environment host for $\mathrm{pH}$ indicators as monolithic disks or thin films with excellent optical properties. Sol-gel technology is convenient to the development of optical $\mathrm{pH}$ sensors. It allows low temperature preparation of optically transparent porous thin films with tunable properties. There is a great potential use of the sol-gel glasses encapsulated materials for optical sensing because of their optical transparency and their mechanical and thermal stability. In recent years, an increased research effort has been focused on doped porous sol-gel layers coated on glass in order to perform fiber optical chemical and biochemical sensing [1-5]. The formation of mesostructured silica formed by cooperative self-assembly of silicate and surfactants was used to modify its structures [6,7]. More recently, organic functional groups were introduced in inorganic precursors, and their copolymerization with tetraethylorthosilicate (TEOS) results in inorganic/organic hybrid

* Correspondence: issanahhal@hotmail.com

Chemistry Department, Al-Azhar University of Gaza, Gaza 1277, Palestine materials $[8,9]$. In addition to using different organically modified precursors, film properties can be tuned with surfactants to have an effect on film morphology and porosity of encapsulated reagents $[10,11]$. Optimization of the glass composition and the right choice of processing parameters are vital for obtaining high quality films that will retain the required sensing performance characteristics. Three methods were used for immobilization of indicators into sol-gel glasses, including impregnation, covalent binding and chemical doping. In a previous work, chemical doping method was used for the immobilization of bromothymol blue (BTB). The synthetic procedure has been designed to obtain monolithic disks of doped BTB in silica $[12,13]$. The role of the cationic cetyltrimethylammonium bromide (CTAB) and the zwitterionic dimethyl amino oxide (GLA) surfactants in modification of the polysiloxane structure network was examined [14]. In this work, optically transparent solgel thin films containing encapsulated BTB molecules were prepared by depositing a sol mixture containing TEOS, BTB and surfactant onto glass substrate using spin-coating techniques in one-pot synthesis. The immobilized thin film material was prepared by entrapping of BTB molecules in the sol-gel matrices. Matrix morphology was controlled by varying the ratios of sol 
Table 1 Weighing of slides

\begin{tabular}{lccc}
\hline $\begin{array}{l}\text { Number } \\
\text { of layers }\end{array}$ & $\begin{array}{c}\text { Weights of empty } \\
\text { slides (g) }\end{array}$ & $\begin{array}{c}\text { Weights of coated } \\
\text { slides (g) }\end{array}$ & $\begin{array}{c}\text { Weight } \\
\text { difference (g) }\end{array}$ \\
\hline Single & 0.516 & 0.532 & 0.016 \\
Double & - & 0.550 & 0.034 \\
Triple & - & 0.560 & 0.044 \\
\hline
\end{tabular}

mixtures and the surfactants. The effects of the sol-gel mixture on the stability and homogeneity of the films and colorimetric response to $\mathrm{pH}$ solutions were also examined.

\section{Methods}

\section{Materials and apparatus}

TEOS, BTB, CTAB and GLA were purchased from Sigma-Aldrich Corporation (MO, USA). Hydrochloric acid, sodium hydroxide and absolute ethanol (98\%) were purchased from Merck \& Co., Inc. (NJ, USA). All chemicals were used as received without further purifications.

\section{Preparation of thin films}

The optical BTB thin films were prepared by spin coating $(2,000 \mathrm{rpm}, 30 \mathrm{~s})$ of the $0.5 \mathrm{ml}$ solution containing 1:1:1 volume ratio of TEOS, BTB and surfactant with molar ratio $(1.5,0.018$ and 0.16 , respectively) onto glass slide $(2.5 \mathrm{~cm} \times 2.5 \mathrm{~cm} \times 1.0 \mathrm{~mm})$. Prior to film deposition, the glass substrate was activated by treating it with concentrated nitric acid for $6 \mathrm{~h}$. After which, slides were washed with distilled water and dried at $100^{\circ} \mathrm{C}$ and then stored in the darkness at room temperature. Small size slides $(1 \times 0.5 \mathrm{~cm})$ were cut into small strips. The coated strips were immersed in the cuvettes to perform UV-vis

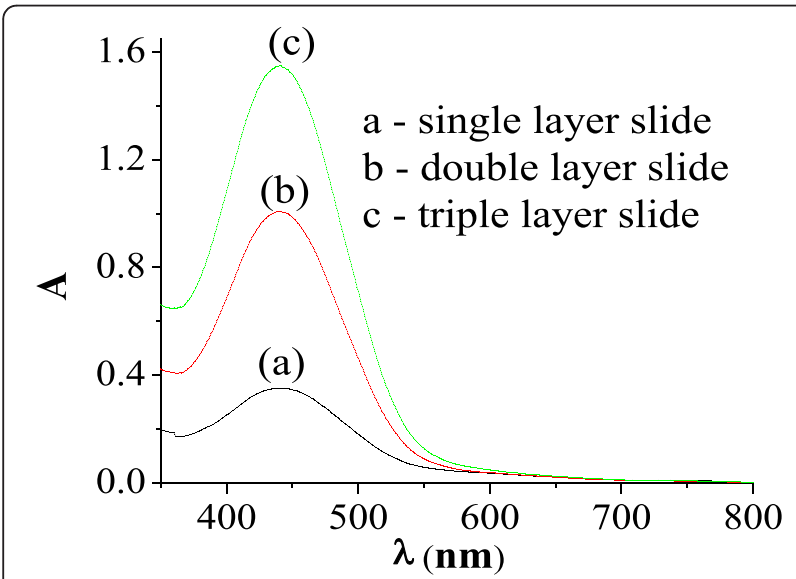

Figure 1 Absorption spectra of immobilized BTB thin film dried at $100^{\circ} \mathbf{C}$. (a) Single layer slide, (b) double layer slide and (c) triple layer slide.

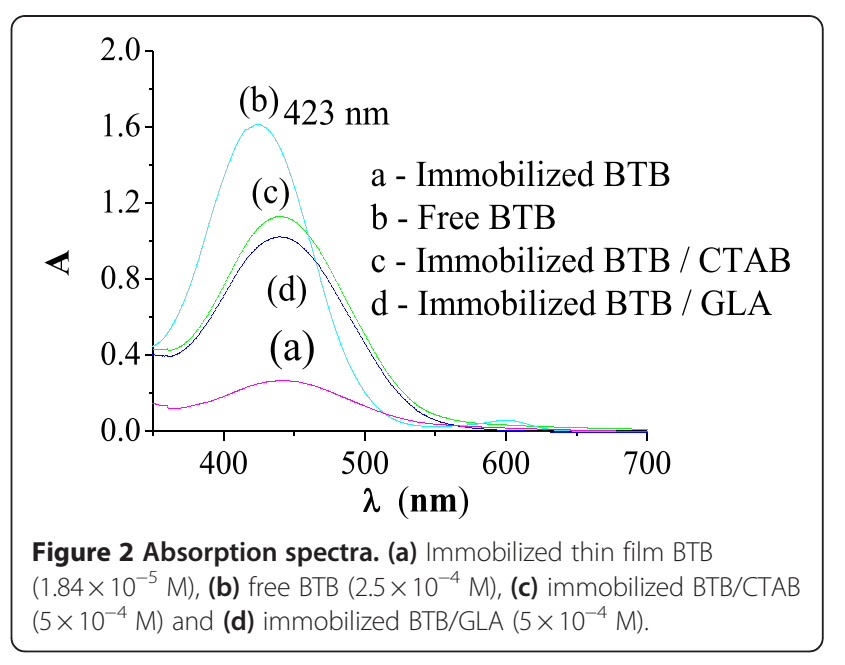

measurements. The obtained wet layer was dried for 5 days at room temperature, then heated for $8 \mathrm{~h}$ at $80^{\circ} \mathrm{C}$, followed by further heating at $100^{\circ} \mathrm{C}$ for $5 \mathrm{~h}$. For obtaining multilayers, the spin-coating method was repeated in the single-layered substrate.

\section{UV-vis spectra}

The optical absorption of the deposited films was obtained using a UV-vis spectrophotometer, Shimadzu1601 (Shimadzu Corporation, Tokyo, Japan), in the range of 200 to $800 \mathrm{~nm}$.

\section{Polarization microscopy}

Surface morphology of the films was characterized using a Zeiss standard polarization microscope (PM) model BX 50 (Carl Zeiss AG, Oberkochen, Germany) equipped with an Olympus DP 100 camera (Olympus Imaging America Inc., PA, USA).

\section{Results and discussion}

Synthesis

Optical BTB thin films were obtained by depositing monolayers of a sol containing TEOS, BTB pH-indicator

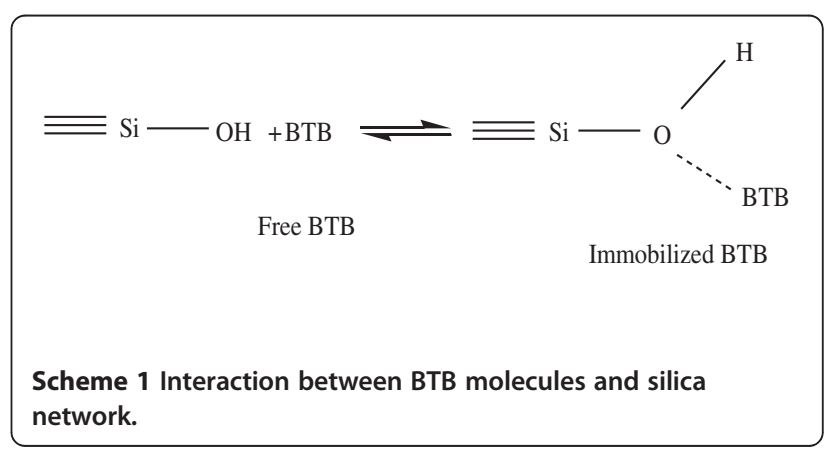




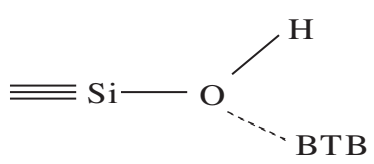

Immobilized BTB
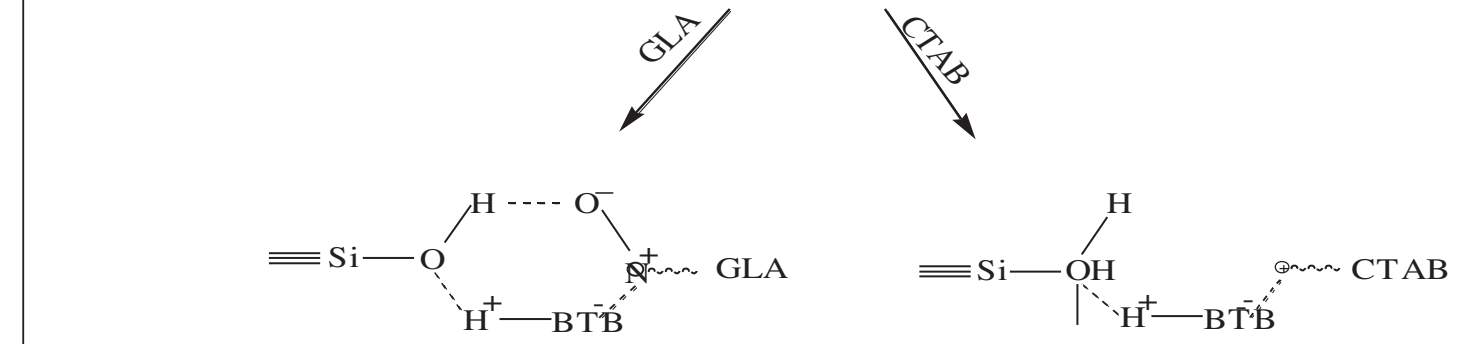

Scheme 2 Immobilized BTB/CTAB \& Immobilized BTB/GLA.

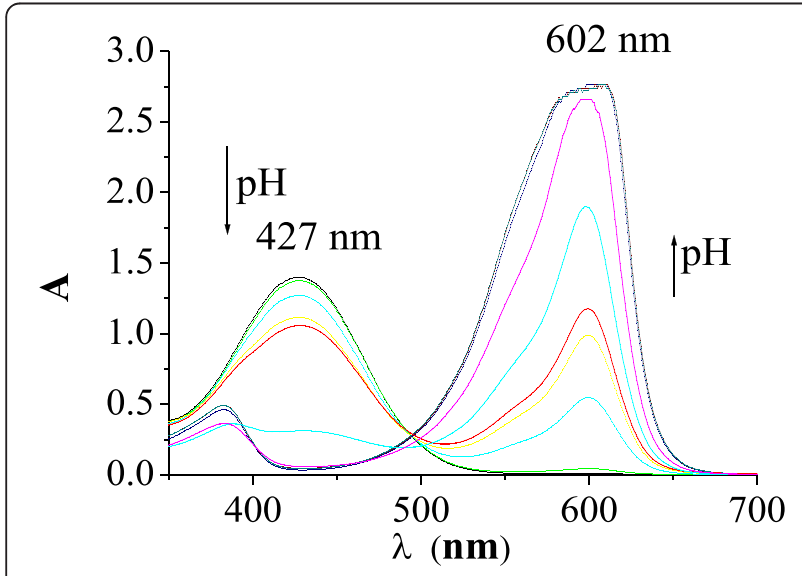

Figure 3 Absorption spectra. Spectra of free BTB $\left(2.5 \times 10^{-4} \mathrm{M}\right)$ in ethanolic solution versus $\mathrm{pH}$ ( $\mathrm{pH}=2$ to 10 at constant time).

and a surfactant into a glass side. It is notable that BTB thin films showed no significant leaching of BTB molecules in the presence of GLA surfactant. This is probably due to the physical interactions between BTB molecules and GLA surfactant. Multilayer coating has been

$\begin{array}{cc}\mathrm{HBTB}^{+} \rightleftharpoons \mathrm{k}_{1} & \mathrm{BTB}^{-}+2 \mathrm{H}^{+} \\ \mathrm{pH}<3 & \mathrm{pH}>8 \\ \text { Acidic form (yellow) } & \text { Basic form (blue) }\end{array}$

Scheme 3 Equilibrium between acid and base forms of BTB.
Table $2 \mathrm{pH}$ response ranges and pKa values for free and immobilized BTB

\begin{tabular}{llll}
\hline & $\mathbf{p K a}_{\mathbf{1}}$ & $\mathbf{p K a}_{\mathbf{2}}$ & $\mathbf{p H}$ response range \\
\hline Free BTB & $6.60(7.0)^{\mathrm{a}}$ & & 4 to 8 \\
Immobilized BTB & $4.84(1.6)^{\mathrm{a}}$ & $10.8(9.7)^{\mathrm{b}}$ & 3 to $7,8.5$ to 12 \\
Immobilized BTB/CTAB & 4.70 & 11.20 & 3 to 7, 8.5 to $1.5 \mathrm{M} \mathrm{[OH]}$ \\
Immobilized BTB/GLA & 4.04 & 11.60 & 3 to 7, 8.5 to 1.5 M [OH] \\
\hline a Reported value in reference [13]; ${ }^{\text {b }}$ reported value in reference [12].
\end{tabular}

deposited in order to obtain thicker coating thin films by repeating the coating process (spinning, drying and densification). The thickness of the layers increases practically linearly with increasing the number of layers. This has been proved by weighting the slide with and without coating (Table 1). The variation of the absorbance band at $440 \mathrm{~nm}$ of the immobilized BTB thin film of different layers is shown in Figure 1. The absorbance increases as the number of layers is increased.

\section{UV-vis spectra}

At normal condition, BTB exists in two forms: acid form (yellow color) and base form (blue color) which
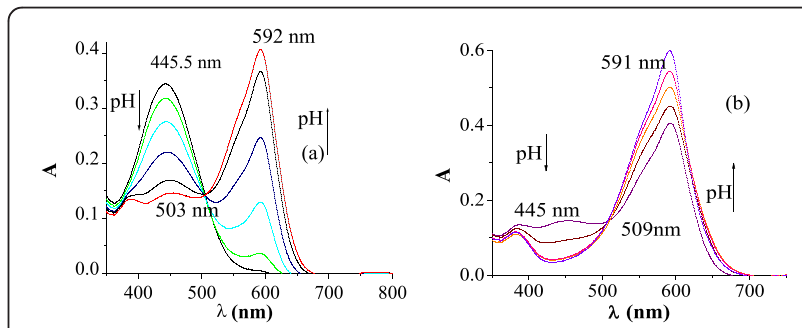

Figure 4 Absorption spectra of immobilized BTB $\left(1.84 \times 10^{-5} \mathrm{M}\right)$

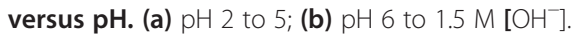




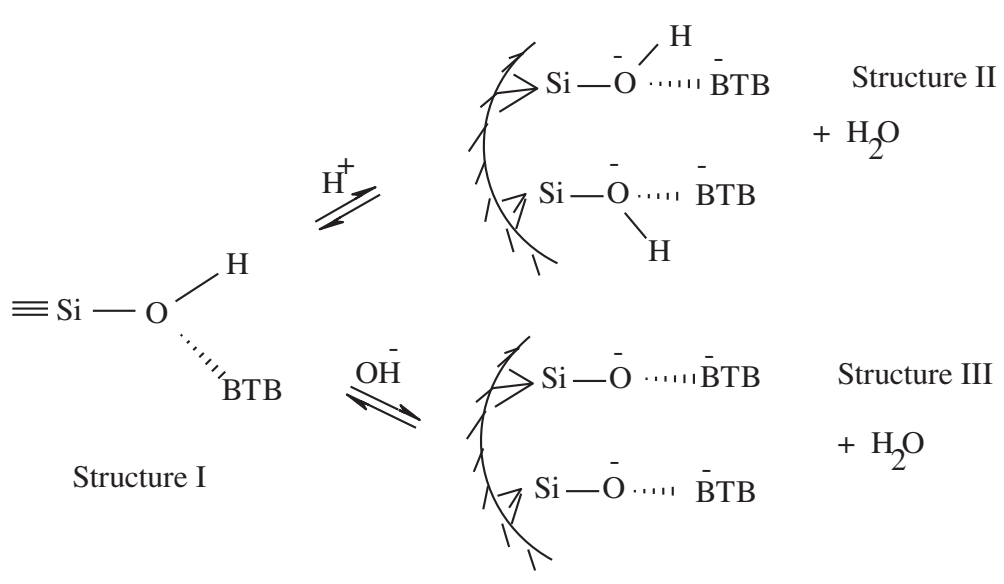

Where …ul: rearangement

Scheme 4 Behavior of immobilized BTB in neutral, acidic and basic media.

correspond to the two absorption bands at about 423 and $602 \mathrm{~nm}$, respectively. These peaks are due to $\pi-\pi^{*}$ and $\mathrm{n}-\pi^{*}$ transitions. The absorption spectrum of the free BTB $\left(2.5 \times 10^{-4} \mathrm{M}\right)$ before exposing to $\mathrm{pH}$ effect is almost similar to that of the immobilized BTB $(1.84 \times 10$ $\left.{ }^{-} 5 \mathrm{M}\right)$ as shown in Figure 2. One absorption band was observed at $423 \mathrm{~nm}$ for the free BTB in solution (Figure 2b), whereas an absorption band was observed at $440 \mathrm{~nm}$ with a red shift of $k 17 \mathrm{~nm}$ for the immobilized BTB (Figure 2a). This could be explained as a result of weak physical interaction between BTB molecules and silica network (Scheme 1) [12-15].

The electronic spectrum of the immobilized BTB (Figure $2 \mathrm{~b}$ ) is very similar to that of the immobilized BTB/CTAB (Figure 2c) and the immobilized BTB/GLA (Figure $2 \mathrm{~d}$ ) when CTAB and GLA surfactants were used. They show no significant shift of the absorption band at $440 \mathrm{~nm}$ but increase the intensity of peak significantly. The reason for this behavior is that CTAB or GLA surfactants have modified the mesostructure of the BTB thin film material, and therefore, low leaching of the BTB molecules was observed (Scheme 2). This is more pronounced in the case of GLA thin film.

The absorption spectra of the free BTB at different $\mathrm{pH}$ values were shown in Figure 3 with the presence of one indicating transition at $\mathrm{pH} 2$ to 10 [12-15]. This transition was due to the change from yellow color (acid form) to blue color (base form) with the presence of one isosbestic point at $492 \mathrm{~nm}$. Dissociation constant (pKa) value was found to be 6.6 which is slightly lower from the reported pKa 7.0 [15] as shown in Scheme 3

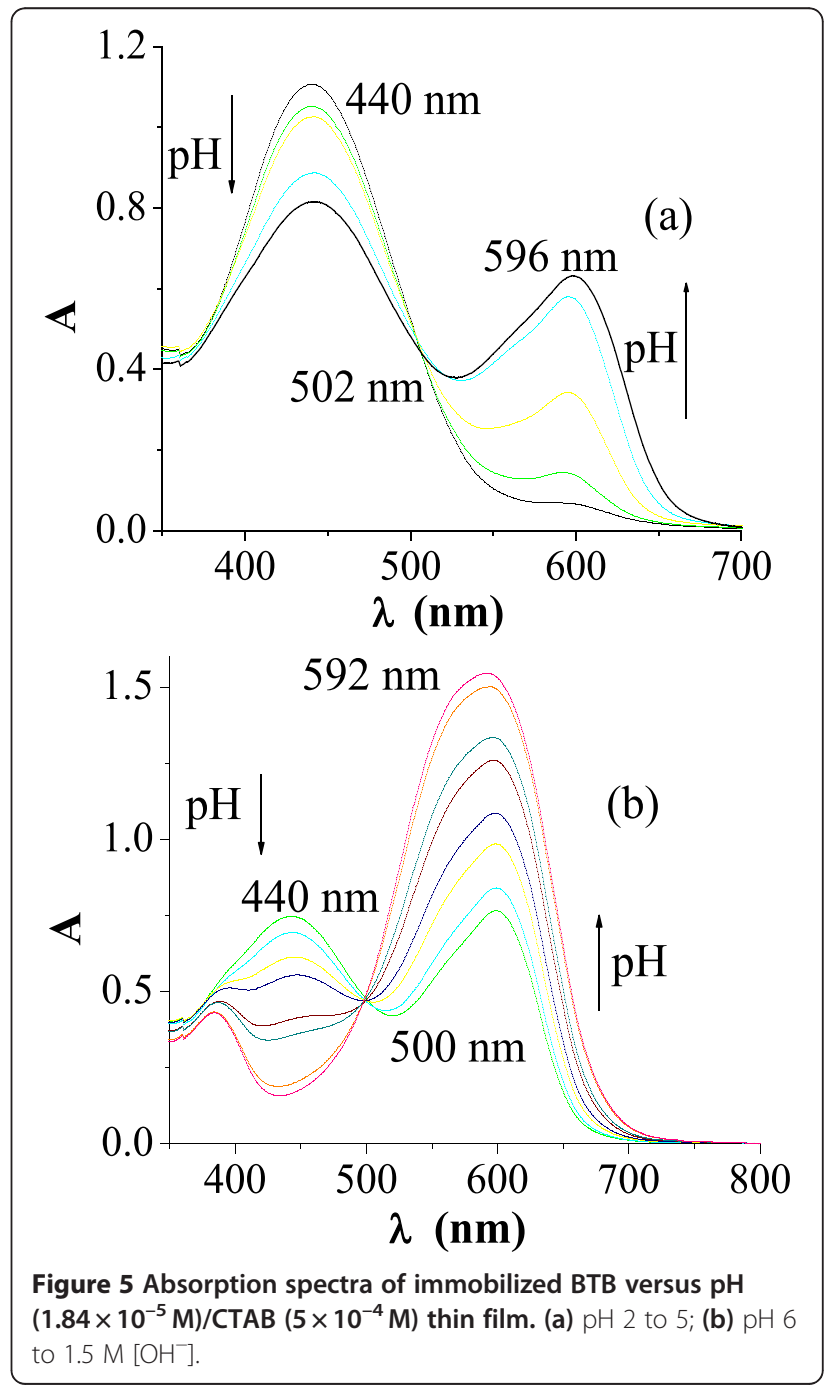




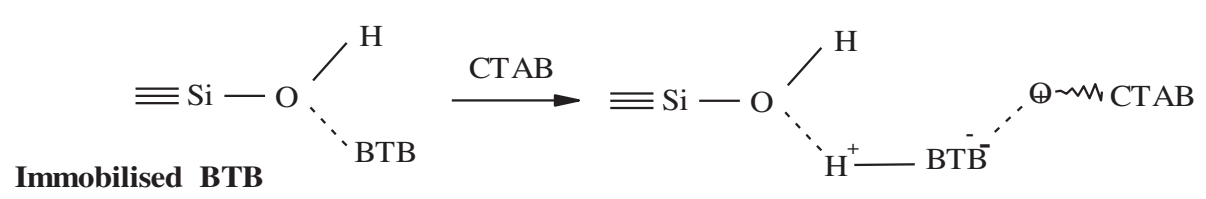

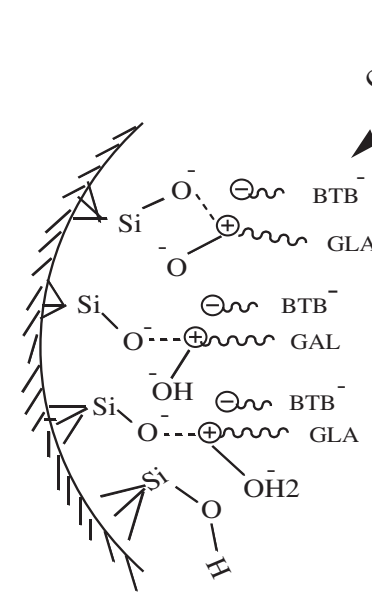

Structure II

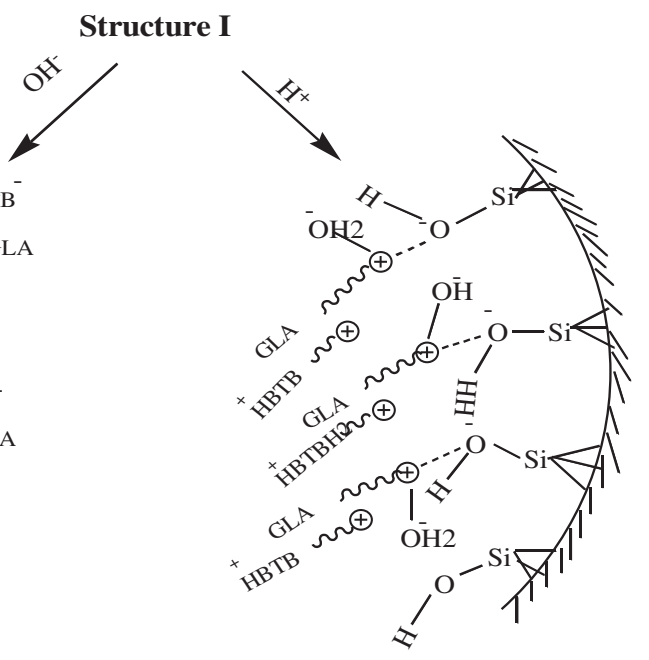

Structure III

Scheme 5 Behavior of immobilized BTB/CTAB in neutral, acidic and basic media.

(Table 2). pKa values were calculated from the titration curves.

When the immobilized BTB is treated with different $\mathrm{pH}$ solutions ( $\mathrm{pH} 2$ to 12), two indicating transitions for free BTB were clearly shown in Figure 4a,b with the presence of two isosbestic points at 503 and $509 \mathrm{~nm}$, respectively, which are consistent with reported results of similar systems $[14,15]$. This provides evidence for the presence of two equilibriums: the first equilibrium at $\mathrm{pH}$ 2 to 7 is between acid and neutral forms of BTB (Figure 4a); the second equilibrium at pH 7 to 12 is between neutral and base forms (Figure $4 \mathrm{~b}$ ). $\mathrm{Pka}_{1}$ and $\mathrm{pka}_{2}$ values for the immobilized BTB were found to be 4.84 and 10.8 , respectively (Table 2 ). There was a considerable difference between our pKa values and the reported ones, in particular, $\mathrm{pKa}_{1}$ value (Table 2) [15]. The lower value of $\mathrm{pka}_{1}, 4.84$, is perhaps due to a weak Van der Waals interaction between protonated $\left(\mathrm{HBTB}^{+}\right)$and silanol species (Structure II of Scheme 4). The higher value of $\mathrm{pKa}_{2}, 10.8$, is probably that, in basic medium, the anions $\mathrm{BTB}^{-}$are probably involve in weak Van der Waals interactions with silanol $\left(\equiv \mathrm{Si}-\mathrm{O}^{-}\right)$species (Structure III of Scheme 4), but in the neutral medium, a hydrogen bonding species ( $\equiv \mathrm{Si}-\mathrm{OH} . . \mathrm{BTB}$ ) was probably formed as described in Structure I of Scheme 4.

When the immobilized BTB/CTAB were treated with different $\mathrm{pH} 2$ to $1.5 \mathrm{M}[\mathrm{OH}]$ solutions, two different indicating transitions (two equilibriums) were also observed (Figure 5a,b), with the presence of isosbestic point for each equilibrium at 502 and $500 \mathrm{~nm}$, respectively. The first transition at $\mathrm{pH} 2$ to 7 was due to the change of yellow color (acid form ) to red color (neutral form) (Figure 5a), and the second transition at $\mathrm{pH} 8$ to $1.5 \mathrm{M}[\mathrm{OH}]$ was due to the change of red color (neutral form) to blue color (base form) (Figure $5 \mathrm{~b}$ ). In the presence of CTAB, the hydrophobic non-charged BTB molecules are transferred to anionic hydrophilic species and formation of ion pairs between the cationic surfactant CTAB and the anionic species of BTB (Structure I of Scheme 5). In the basic medium, ion pairs between cationic CTAB and BTB anions were formed (Structure II of Scheme 5). There is change of $\mathrm{pKa}_{2}$ to more basic in the presence of cationic surfactant CTAB (Table 2). In acidic medium, a weak interaction between the protonated BTB and silanols increases $\mathrm{pKa}_{1}$ to more acidic value (Structure III of Scheme 5). This can be explained that the micellar environment favors the anionic hydrophilic species of BTB as given in Scheme 5.

When the immobilized BTB/GLA is treated with different $\mathrm{pH} 2-1.5 \mathrm{M}[\mathrm{OH}]$ solutions, two different indicating transitions (two equilibriums) are also observed as given in Figure 6a,b with the presence of an isosbestic point for each equilibrium at 504 and $501 \mathrm{~nm}$, respectively. As the $\mathrm{pH}$ increases from 2 to 7 , first equilibrium 


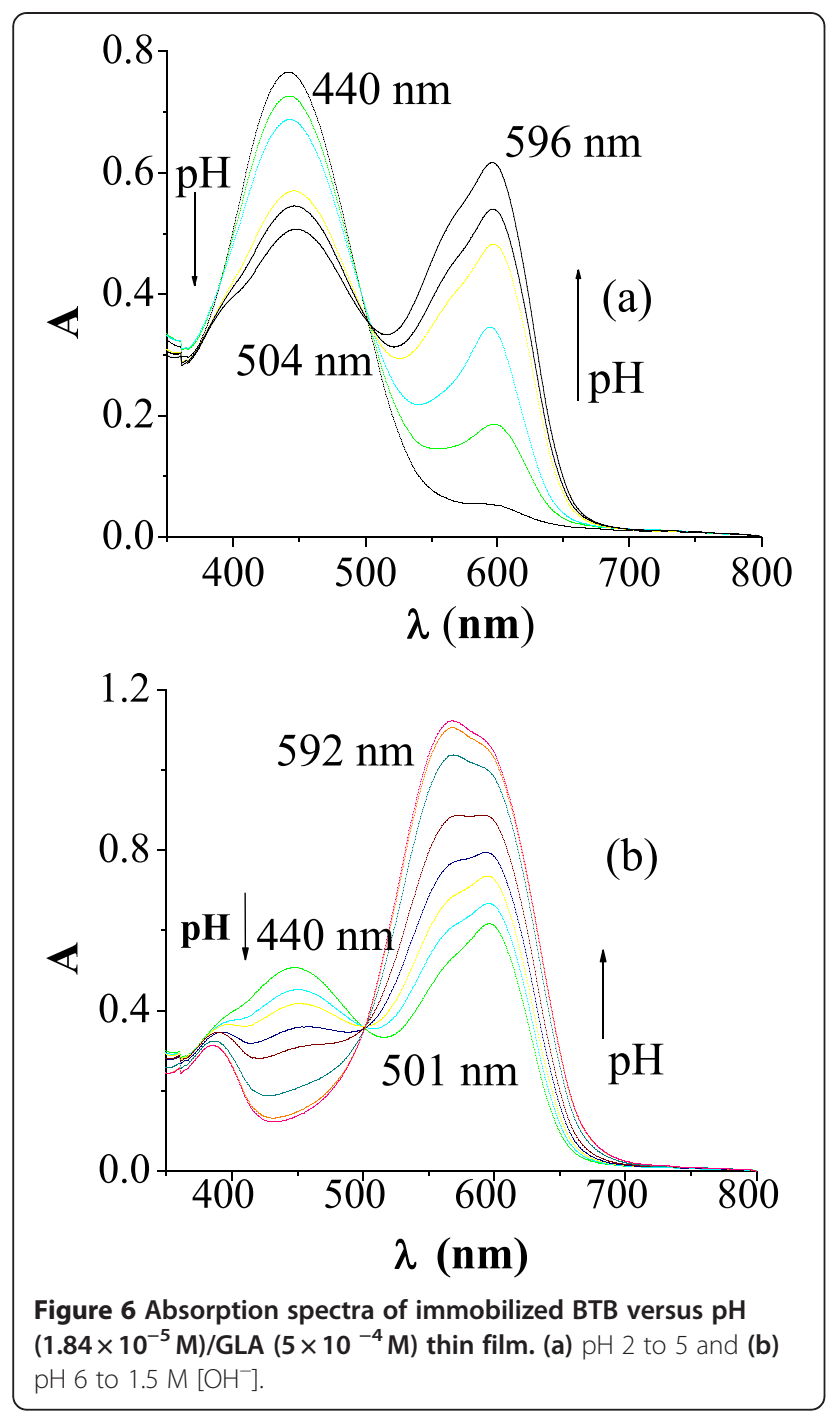

is accompanied with a change from the yellow color (acid form) to the red color (neutral form) (Figure 6a), and as the $\mathrm{pH}$ increases from 8 to $1.5 \mathrm{M}[\mathrm{OH}]$, the second equilibrium is accompanied by a change of the red color (neutral form) to the blue color (base form) as shown in Figure 6b. Due to the formation of ion pairs between GLA surfactant and the anionic species of BTB, there was a faster change from the hydrophobic noncharged BTB molecules into the anionic hydrophilic in the presence of GLA species than that in the presence of CTAB. This is probably because of the strong interaction between both positive and negative charges of the zwitterionic surfactant and both BTB species $\left(\mathrm{BTB}^{-}\right.$and $\left.\mathrm{H}^{+}\right)$ as shown in Structure I of Scheme 6. There was a slight change of $\mathrm{pKa}_{1}$ to more acidic value and $\mathrm{pka}_{2}$ to more basic value in the presence of GLA (Table 2). The nature of interactions at neutral, basic and acidic media is shown in Scheme 6 (Structures I, II and III).

\section{Effect of temperature}

BTB thin films of different layers (single, double and triple) have been dried at different temperatures (RT, $50^{\circ}$ $\mathrm{C}, 80^{\circ} \mathrm{C}$ and $100^{\circ} \mathrm{C}$ ). It has been found that the highest intensity was observed at $440 \mathrm{~nm}$ for samples dried at $100^{\circ} \mathrm{C}$ (Figure 7). This can be explained that the films dried at $100^{\circ} \mathrm{C}$ have evaporated most of water and alcohol molecules which are physically absorbed within the silica pores, and therefore, BTB molecules were better accommodated within the silica pores and strongly interacted within the silica matrix.

\section{The effect of leaching}

It is found that the lowest leaching of BTB molecules from these films was observed when GLA surfactant was used (Figure 8). The reason for this change is due to strong interaction between cation and anion ends of the zwitterionic surfactant with the anion and the proton species of BTB. It is also found that the lowest leaching was found when these films were dried at higher temperature (Figure 7).

\section{Reversibility of the BTB sensor}

BTB thin films act as reversible sensors for the changes of $\mathrm{pH}$, forward and backward as given in Figure 9a,b. The spectra were obtained in the range from 350 to $700 \mathrm{~nm}$; the response time was $20 \mathrm{~min}$ in both ways.

\section{Reproducibility of the BTB sensor}

Using a thin film, six-cycle measurements were conducted. The cycles were repeated every $24 \mathrm{~h}$ from the previous one to check the intra-week reproducibility. It was found that the absorbance after each measurement over the $\mathrm{pH} 5$ to 9 range gradually decreased and reached a minimum value after $144 \mathrm{~h}$ (Table 3). This is probably due to some degradation and significant leaching of BTB species. It is notable that there is no decreasing of absorbance when GLA surfactant was used.

\section{Stability of BTB thin film sensors}

To investigate the stability of the sensors, the sol-gel slides were examined after a period of 10 months. After 10 months, the absorbance spectrum of the film was the same. The thickness of the film response has not changed. This may prove that the microstructure of the film is fully stable after a period of 10 months.

\section{Polarized light microscopy}

In order to examine the nature of the interaction between BTB, silica and surfactants, several photographs were taken by crossed polarized light microscope for thin films of free silica, immobilized BTB, immobilized $\mathrm{BTB} / \mathrm{CTAB}$ and immobilized BTB/GLA. It is clear that the particle size distribution has a wider range in the 


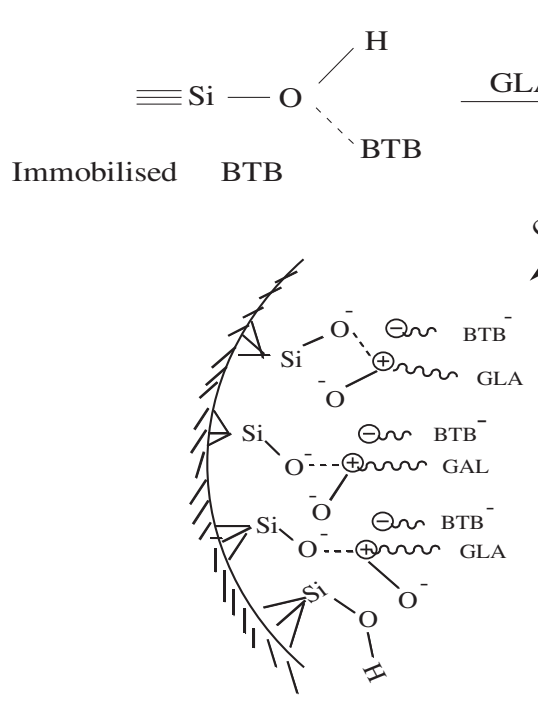

Structure II
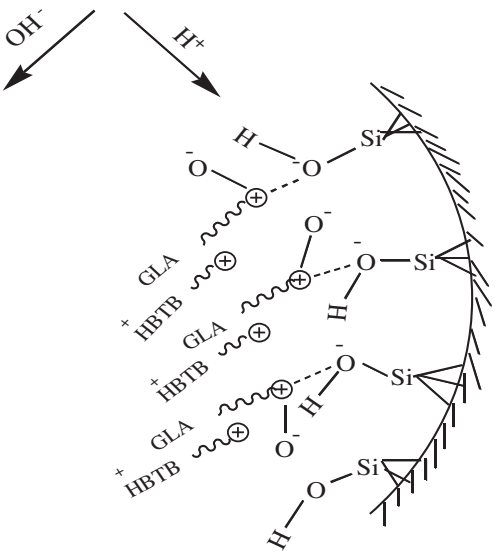

Structure III

Scheme 6 Behavior of immobilized BTB/GLA in neutral, acidic and basic media.

free silica (Figure 10a), whereas more uniform and smaller size particles were found for the immobilized BTB (Figure 10b). Large number of particles and more uniform particles were observed when GLA and CTAB were used (Figure 10c,d). This behavior could be attributed to the interaction between the surfactants with BTB molecules through electrostatic interaction. This was also supported by the low leaching of BTB molecules in the presence of surfactants, where the

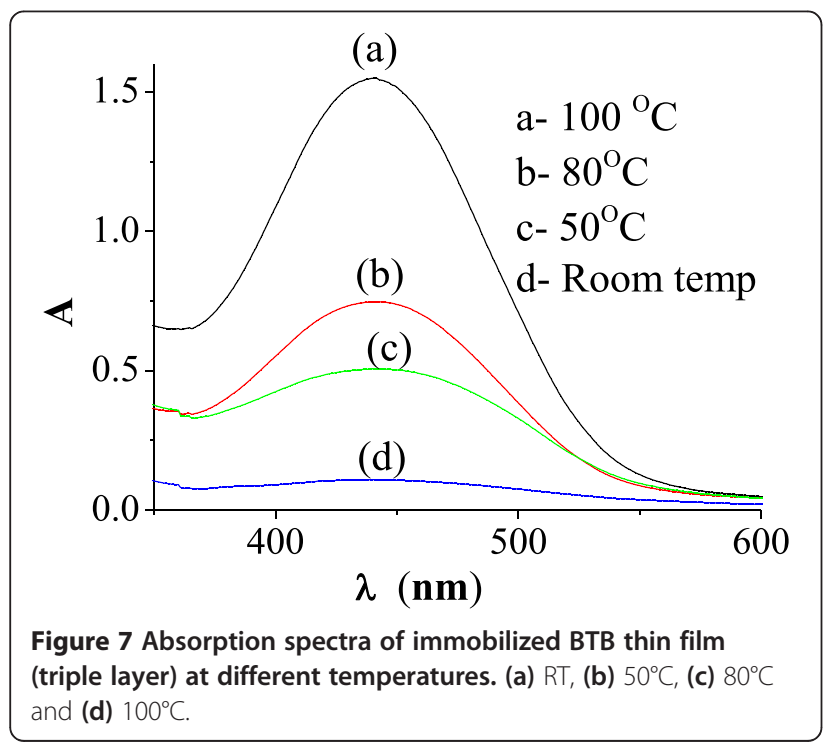

hydrophobic part of surfactant participates in the acceleration of the association of the particles which lead to increase its stability. From Figure 10d, the GLA surfactant has more effect on the improvement and modification of the particles than CTAB (Figure 10c). This behavior may refer to the chemical structure of GLA. There is a strong interaction between the cation and anion ends of GLA with BTB more than CTAB which has a bulky head group (carry a positive charge). Figure 10d shows a smaller and fine sequence of particles.

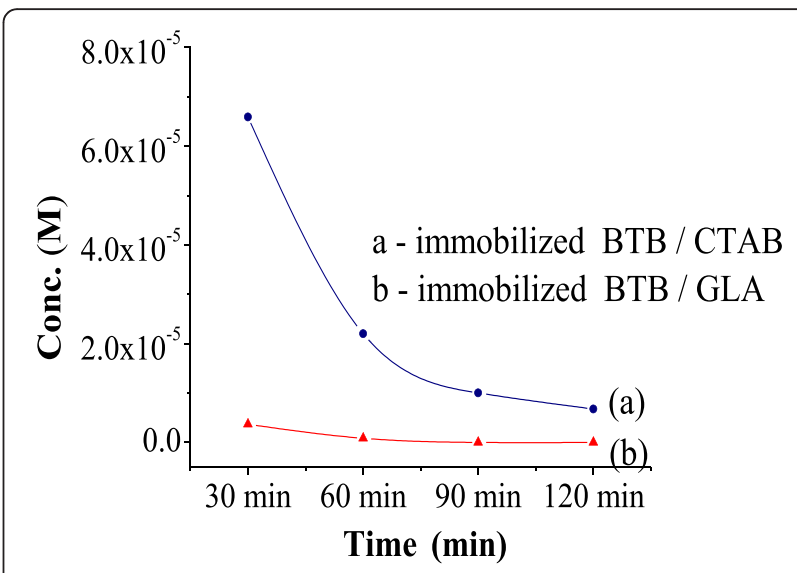

Figure 8 Leaching of BTB (M) thin films versus time (minutes). (a) BTB/CTAB film, (b) BTB/GLA film. 


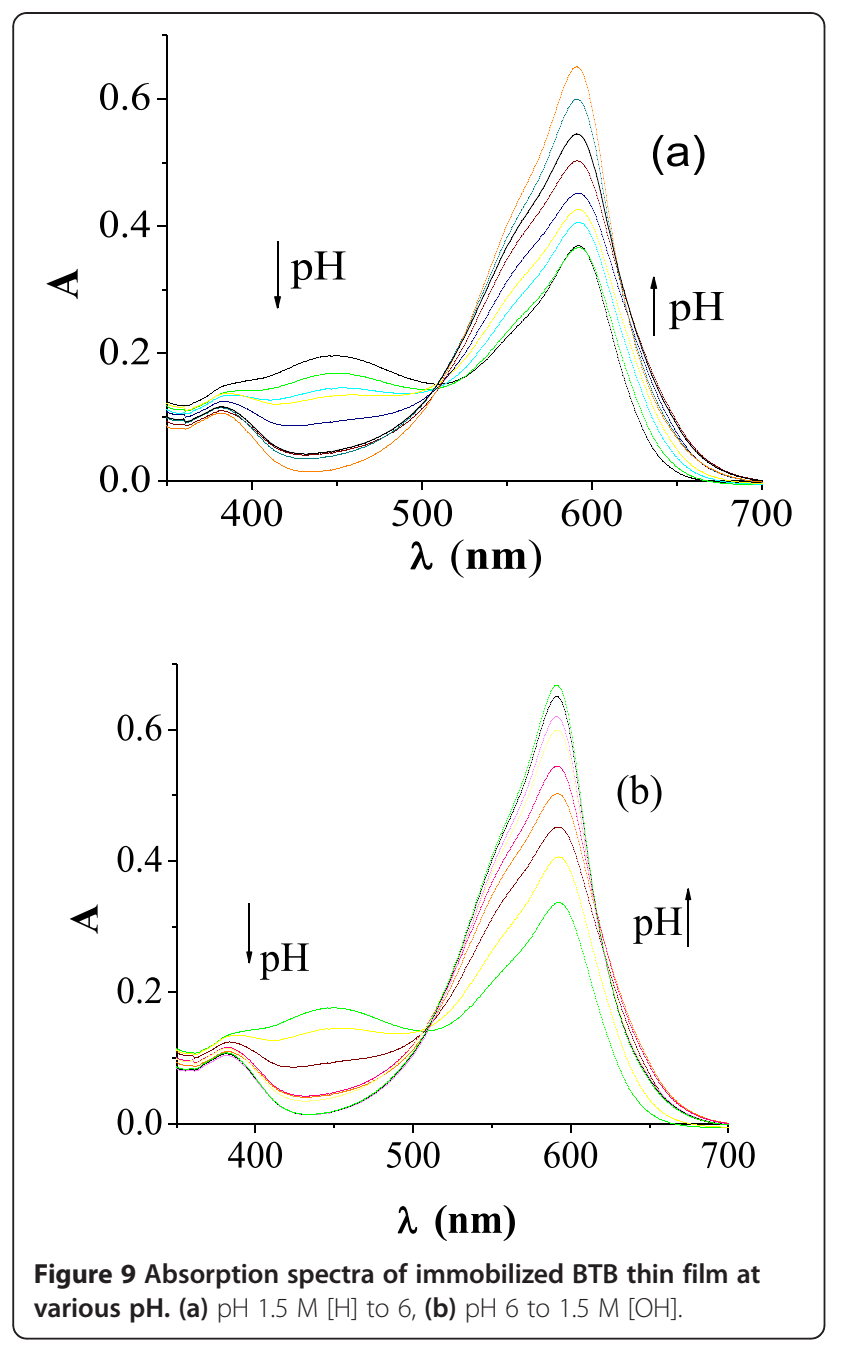

Table 3 Reproducibility of immobilized BTB thin film (single layer) sensors

\begin{tabular}{ccc}
\hline Measurement number & Time testing (h) & Absorbance at $\mathbf{5 9 2} \mathbf{~} \mathbf{m}$ \\
\hline 1 & 0. & 0.453 \\
2 & 24 & 0.427 \\
3 & 48 & 0.415 \\
4 & 72 & 0.391 \\
5 & 96 & 0.365 \\
6 & 120 & 0.334 \\
7 & 144 & 0.210 \\
\hline
\end{tabular}

\section{Conclusion}

Three different BTB thin films, immobilized BTB, immobilized BTB/CTAB and immobilized BTB/GLA were prepared by depositing a thin layer of a solution containing TEOS, BTB and CTAB or GLA surfactant onto glass substrate. The lowest leaching of BTB species was obtained when GLA surfactant was used. BTB molecules were properly accommodated within silica network pores when the thin films were heated at $100^{\circ} \mathrm{C}$ and well interacted when surfactants were used. These sensors were found to be very sensitive for $\mathrm{pH}$ change with the presence of two indicating transitions. The presence of surfactants has also improved silica network and increased its surface area.

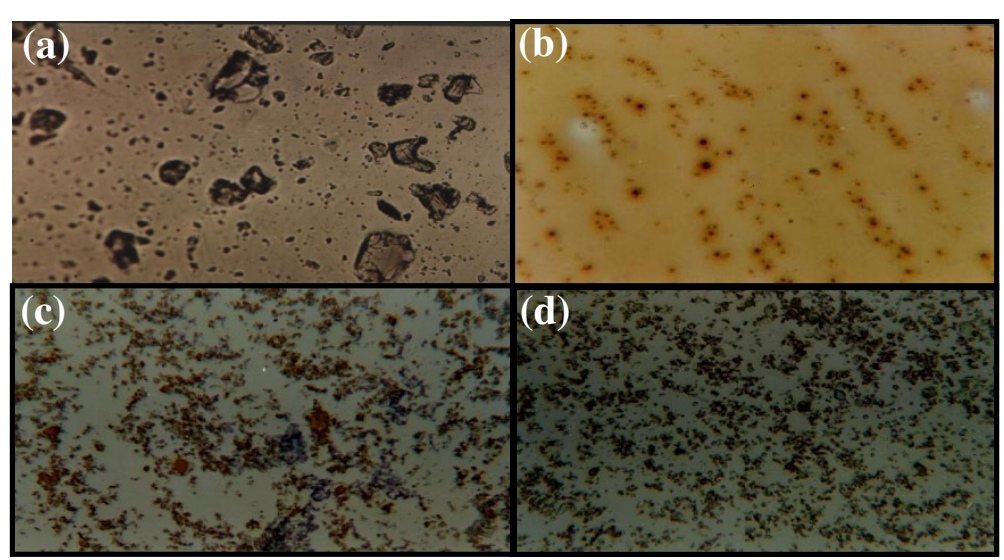

Figure 10 Polarized light microscope photographs. (a) Free silica, (b) immobilized BTB, (c) immobilized BTB/CTAB and (d) immobilized BTB/GLA. 


\section{Competing interests}

The authors declare that they have no competing interests.

\section{Authors' contributions}

$A Q$ and FK carried out the preparations of coating immobilized BTB thin films and conducted the UVNIS spectra. IN and SZ participated in the discussions and interpretation of all spectroscopic results. All authors read and approved the final manuscript.

\section{Authors' information}

IN was born in Gaza in 1956. He had his PhD degree from Manchester University, UK in 1986. He is a professor of Inorganic Chemistry at Al-Azhar University of Gaza. He has many publications in several topics, especially in immobilized polysiloxane ligands and their applications. SZ was born in Gaza in 1948. He had his PhD degree from Al-Exandria University, Egypt in 1978. He is a professor of Physical Chemistry at Al-Azhar University of Gaza. He has many publications in several topics, especially in colloidal chemistry fields and their applications. FK is a PhD student, and AQ is masters degree student.

\section{Acknowledgments}

The authors would like to thank Al-Azhar University of Gaza and the Chemistry Department at AUG for providing their laboratory facilities for completing this research.

Received: 16 March 2011 Accepted: 28 February 2012

Published: 18 July 2012

\section{References}

1. Zusman, R, Rottman, C, Ottolenghi, M, Avnir, D: Doped sol-gel glasses as chemical sensors. J. Non-Cryst. Solids 122, 107 (1990)

2. Wolfbels, OS: Fiber-optic chemical sensors and biosensors. Anal. Chem. 80, 4269 (2008)

3. Jeronimo, PCA, Araujo, AN, Conceição, M, Montenegro, BSM: Optical sensors and biosensors based on sol-gel films review article. Talanta 72, 13 (2007)

4. VonBultzingslowen, C, McEvoy, AK, McDonagh, C, MacCraith, BD, Klimant, I, Krause, C, Wolfbeis, OS: Sol-gel based optical carbon dioxide sensor employing dual luminophore referencing for application in food packaging technology. Analyst 127, 1478 (2002)

5. Chodavarapu, VP, Bukowski, RM, Titus, AH, Cartwright, AN, Bright, FV: CMOS integrated luminescence oxygen sensor system. Electron. Lett. 43, 688 (2007)

6. Miled, OB, Grosso, D, Sanches, C, Livage, J: An optical fibre pH sensor on dye doped mesostructured silica. J. Phys. Chem. Solids 65, 1751 (2004)

7. Jurmanovic, S, Kordic, S, Steinberg, MD, Steinberg, IM: Organically modified silicate thin films with colorimetric $\mathrm{pH}$ indicators methyl red and bromocresol green as $\mathrm{pH}$ responsive sol-gel hybrid material. Thin Solid Films 518, 2234 (2010)

8. Lev, O, Tsionsky, M, Rabinovich, L, Glezer, V, Sampath, S, Pankratov, I, Gun, J: Organically modified sol-gel sensors. Anal. Chem. 67, 22A (1995)

9. Dash, S, Mishra, S, Patel, S, Misshra, BK: Organically modified silica: synthesis and applications due to its surface interaction with organic molecules. Adv. Colloid Interface Sci. 140, 77 (2008)

10. Lu, YF, Ganguli, R, Drewien, CA, Anderson, MT, Brinker, CJ, Gong, WL, Guo, YX, Soyez, H, Dunn, B, Huang, MH, Zink, Jl: Continuous formation of the supported cubic and hexagonal mesoporous films by sol-gel dip coating. Nature 389, 364 (1997)

11. Rottman, C, Grader, G, De Hazan, Y, Melchior, S, Avnir, D: Surfactant-induced modification of dopents in sol-gel matrixes. J. Am. Chem. Soc. 121, 8533 (1999)

12. El Nahhal, IM, Zourab, SM, Quidah, FS: Behaviour of trapped bromothymol blue into sol-gel matrix in presence of CTAB. J Dispersion Sci Technol 30, 379 (2009)

13. Zaggout, FR, J: Encapsulation of bromothymol blue pH indicator into solgel matrix. Dispersion Sci. Technol. 27, 175 (2006)
14. El Nahhal, IM, Zourab, SM, Quidah, FS, J: Encapsulation of bromothymol blue into a polysiloxane network matrix in the presence of surfactants. Dispersion Sci. Technol 32, 1367 (2011)

15. Safavi, A, Maleki, N, Bagheri, M: Modification of chemical performance of dopants in xerogel films with entrapped ionic liquid. J. Mater. Chem. 17, 1674 (2007)

doi:10.1186/2228-5326-2-16

Cite this article as: Nahhal et al:: Thin film optical BTB pH sensors using sol-gel method in presence of surfactants. International Nano Letters 2012 2:16.

\section{Submit your manuscript to a SpringerOpen ${ }^{\circ}$ journal and benefit from:}

- Convenient online submission

- Rigorous peer review

- Immediate publication on acceptance

- Open access: articles freely available online

- High visibility within the field

- Retaining the copyright to your article

Submit your next manuscript at springeropen.com 